\title{
Effect of Plasma Subsonic Toroidal Flows Induced by Alfvén Waves on Transport Processes in the Edge of Elongated Tokamaks
}

\author{
V. S. Tsypin, J. H. F. Severo, I. C. Nascimento, \\ R. M. O. Galvão, and Yu. K. Kuznetsov \\ Institute of Physics, University of São Paulo, \\ Rua do Matão, Travessa R, 187, 05508-900, São Paulo, Brasil
}

Received on 4 May, 2000

\begin{abstract}
There is a renewed interest in using Alfvén waves (AW) in tokamak plasmas. Previously, AW were actively explored mostly for current drive and plasma heating in tokamaks. Presently, the possibility of the anomalous and neoclassical transport suppression by AW in tokamak plasmas is being vividly discussed. AW can also induce poloidal and toroidal plasma rotation. Toroidal plasma rotation can reach the subsonic level. These flows can substantially affect neoclassical transport both in collisional and weakly collisional plasmas. In this paper, the effect of plasma subsonic toroidal flows induced by Alfvén waves on transport processes in the edge of elongated tokamak is investigated. The dependence of poloidal plasma rotation and ion heat conductivity on the elongation parameter and the ratio of induced toroidal velocity to the sonic speed are analytically obtained.
\end{abstract}

\section{Introduction}

It is well-known that Alfvén waves are one of the basic methods of current drive (AWCD) and plasma heating (AWPH) in tokamaks. ${ }^{1-5}$ These methods were clearly demonstrated in numerous experiments ${ }^{6-8}$ and will be anew applied in forthcoming experiments in TCABR (Tokamak Chauffage Alfvén Brasil) tokamak. ${ }^{9,10}$ The comparatively recent applications of AW in tokamaks are creation of transport barriers and suppression of anomalous and neoclassical transport by these waves..$^{11-14}$ The main idea is to induce a sheared radial electric field that can take apart the turbulent plasma eddies and thus suppress anomalous transport. ${ }^{15-17}$ Suppression of neoclassical transport in weakly collisional tokamak plasmas is connected with ion banana orbit squeezing by the sheared radial electric field. ${ }^{18,19}$ Alfvén waves can be used to efficiently induce the strongly sheared radial electric field ${ }^{11-14}$ thus achieving suppression of anomalous and neoclassical transport. The first experimental evidence that AW can be useful to create plasma rotation, and, consequently, the radial electric field, was obtained in the Phaedrus-T tokamak. ${ }^{20}$

Besides these very attractive topics, there is also the additional physical mechanism where AW can play a substantial role, i.e., the ion neoclassical transport in the tokamak edge (collisional) plasma. The importance of this mechanism was emphasized in the papers by Rogister, ${ }^{21-23}$ where the experimental results in modern tokamaks were carefully analyzed. Rogister considers the transport processes in tokamak edge plasmas to be in the $\mathrm{H}$-mode (the so-called high regime), when the characteristic length of plasma macroscopic parameters $L_{n}$ is of the order of the ion Larmor radius, $\rho_{i \theta}=c v_{T i} M_{i} / e_{i} B_{\theta p}$, calculated on the poloidal magnetic field $B_{\theta p}$, i.e., $L_{n} \sim \rho_{i \theta}$. Here $e_{i}$ and $M_{i}$ are the ion charge and mass, respectively, $B_{\theta p}$ is the "physical" component of the magnetic field, to differ it from covariant or contravariant components that appear below, and $v_{T i}=\sqrt{2 T_{i} / M_{i}}$ is the ion thermal velocity. However, there are some relevant problems that need to be investigated in collisional tokamak plasmas, in the Lmode (the low regime), when the condition $L_{n}>\rho_{i \theta}$ is fulfilled.

The ion neoclassical transport in tokamak collisional plasmas ${ }^{24}$ was originally investigated by Shafranov. He used the well-known approach by Pfirsch and Schlüter to calculate the ion heat conductivity in tokamak plasmas with circular magnetic surfaces. These results were later generalized for arbitrary geometry of the magnetic field. ${ }^{25}$ The next step in this direction was taken by Mikhailovskii and Tsypin, ${ }^{26}$ who considered the effect of sonic toroidal flows on transport processes in collisional plasmas. The very interesting effect of changing the sign of the ion poloidal velocity at a specific 
value of the ratio of toroidal velocity $U_{i \zeta}$ to sound velocity $c_{s}^{2}=\left(T_{e}+T_{i}\right) / M_{i}$, and the importance of the collisional parameter $b=q^{2} R^{2} / \lambda_{i}^{2}$ were brought up in this paper. Here, $q$ is the inverse rotational transform, $R$ is the tokamak major radius, $\lambda_{i}=v_{T i} / \nu_{i}$ is the ion mean free path, $T_{e}$ and $T_{i}$ are the electron and ion temperature, respectively, and $\nu_{i}$ is the ionion collision frequency. ${ }^{27}$ This study was later continued to determine also the ion heat conductivity taking into account of large values of the collisional parameter $b, 1<b<M_{i} / M_{e},{ }^{28,29}$ external forces, ${ }^{30}$ and elongation. ${ }^{31}$ In this paper we carry on this investigation studying the effect of plasma subsonic toroidal flows induced by Alfvén waves on poloidal plasma rotation and ion heat conductivity in collisional plasmas of elongated tokamaks, with smooth profiles of macroscopic plasma parameters, $L_{n}>\rho_{i \theta}$.

\section{Magnetic field and metric}

To solve our problem, we need to choose the coordinate system to carry out the calculations for a toroidal plasma column with elliptic cross-section. As the calculations of the metrics have been repeatedly presented in many previous publications, we here show only main steps to obtain it. Initially, we take the coordinates $\rho^{\prime}$, $\theta^{\prime \prime}$ and $\zeta$ to be attached to the geometrical center of the magnetic surfaces, where $\rho^{\prime}$ is the radial coordinate in the tokamak cross-section and $\theta^{\prime \prime}$ and $\zeta$ are the poloidal and toroidal angles, respectively. The length element in these coordinates is

$$
d l^{2}=d \rho^{2}+\rho^{2} d \theta^{\prime \prime 2}+\left(R-\rho^{\prime} \cos \theta^{\prime \prime}\right)^{2} d \zeta^{2} .
$$

The next step is the "encircling" of the magnetic surfaces by means of the transformation

$$
\rho \cos \theta^{\prime}=\exp (\eta / 2) \rho^{\prime} \cos \theta^{\prime \prime},
$$

$$
\rho \sin \theta^{\prime}=\exp (-\eta / 2) \rho^{\prime} \sin \theta^{\prime \prime},
$$

where $\rho=\sqrt{l_{1} l_{2}}, \eta=\ln \left(l_{2} / l_{1}\right)$, and $l_{2}$ and $l_{1}$ are semimajor and semiminor axes, respectively, of the magnetic surface. Then, we choose new coordinates to be attached to the geometric center of the tokamak crosssection, and straighten the magnetic lines of force:

$$
\begin{gathered}
\rho \cos \theta^{\prime}=r \cos [\theta+\delta(\theta, r)]+\Delta(r), \\
\rho \sin \theta^{\prime}=r \sin [\theta+\delta(\theta, r)],
\end{gathered}
$$

where $\Delta(r)$ is the shift of the geometric center of the magnetic surface, determined from equilibrium conditions.

The straightening parameter $\delta$ can be found from the condition that the radial component of the current density vanishes, $j^{r}=0$, by taking into account the expression for the magnetic field with the straight lines of force,

$$
\overrightarrow{B_{0}}=\left\{0 ; \quad \chi^{\prime} / 2 \pi \sqrt{g} ; \quad \phi^{\prime} / 2 \pi \sqrt{g}\right\},
$$

(here $\chi$ and $\phi$ are the poloidal and toroidal magnetic fluxes, respectively, and $g$ is the metric tensor determinant), and after finding the metric tensor components $g_{i k}$, after the substitution of Eqs. (2) and (3) into Eq. (1),

$$
\partial\left(g_{33} / \sqrt{g}\right) / \partial \theta=0 .
$$

As a result, the parameter $\delta$ is equal to

$$
\delta=-\sin \theta\left(\varepsilon^{*}+\Delta^{\prime} \exp (\eta / 2)\right) .
$$

Then, we find the metric covariant tensor components and their determinant

$$
\begin{gathered}
g_{11}=\cosh \eta-\sinh \eta \cos 2 \theta+2 \Delta^{\prime} \exp (-\eta / 2) \cos \theta, \\
g_{22}=r^{2}(\cosh \eta+\sinh \eta \cos 2 \theta)\left[1-2 \cos \theta\left(\varepsilon^{*}+\Delta^{\prime} \exp (\eta / 2)\right)\right], \\
g_{12}=g_{21}=r\left\{\sinh \eta \sin 2 \theta-\sin \theta\left[\cosh \eta\left(\varepsilon^{*}+r \Delta^{\prime \prime} \exp (\eta / 2)\right)+\Delta^{\prime} \exp (-\eta / 2)\right]\right\}, \\
g_{33}=R^{2}\left(1-2 \varepsilon^{*} \cos \theta\right), \quad \sqrt{g}=r R\left(1-2 \varepsilon^{*} \cos \theta\right),
\end{gathered}
$$

where the parameter $\varepsilon^{*}$ is $\varepsilon^{*}=\varepsilon \exp (-\eta / 2)$, and $\varepsilon=r / R$ is the tokamak inverse aspect ratio. The angle dependence of the magnetic field can be obtained from the expression $B_{0}=\sqrt{g_{i k} B^{i} B^{k}}$; we have

$$
B_{0}=B_{s}\left[1+\varepsilon^{*} \cos \theta+\left(A \varepsilon^{*} / 2\right) \cos ^{2} \theta\right],
$$

where

$$
A=\frac{\varepsilon^{*}}{q^{2}}[\exp (2 \eta)-1]=\frac{l_{1}}{R q^{2}}\left(\frac{l_{2}^{2}}{l_{1}^{2}}-1\right),
$$

and $q=\phi^{\prime} / \chi^{\prime}$ is the inverse rotational transform defined in general geometry.

We also need a component of the Cristoffel's symbol

$$
\Gamma_{k l}^{i}=g^{i m} \Gamma_{m, k l}
$$




$$
\Gamma_{m, k l}=\frac{1}{2}\left(\frac{\partial g_{m k}}{\partial x^{l}}+\frac{\partial g_{m l}}{\partial x^{k}}-\frac{\partial g_{k l}}{\partial x^{m}}\right),
$$

namely,

$$
\Gamma_{\zeta \theta}^{\zeta}=\varepsilon^{*} \sin \theta
$$

The non-orthogonal coordinates are $x^{i}=(r, \theta, \zeta)$.

\section{Starting equations}

As we mentioned in the Introduction, our main goal is to calculate the surface averaged ion heat flux $\Gamma_{T i}$ and the ion poloidal rotation velocity $U_{i \theta}$ as functions of the ratio $\alpha=U_{i \zeta}^{2} / c_{s}^{2}$ (the squared Mach number) and the elongation parameter $A$. The ion heat flux is defined by the expression

$$
\Gamma_{T i} \approx\left\langle q_{i}^{r}\right\rangle
$$

where

$$
\langle\ldots\rangle=\int_{0}^{2 \pi}(\ldots) \sqrt{g} d \theta / \int_{0}^{2 \pi} \sqrt{g} d \theta
$$

and the heat flux radial contravariant component $q_{i}^{r}$ is equal to

$$
q_{i}^{r}=-\frac{2 p_{i} \nu_{i} g^{11}}{M_{i} \omega_{c i}^{2}} \frac{\partial T_{i}}{\partial r}-\frac{5}{2} \frac{p_{i} g_{33} h^{\zeta}}{M_{i} \omega_{c i} \sqrt{g}} \frac{\partial T_{i}}{\partial \theta} .
$$

Here, $p_{i}$ is the ion isotropic pressure, $\omega_{c i}=e_{i} B / c M_{i}$ is the ion cyclotron frequency, $g^{11}$ is the contravariant component of the metric tensor, and $h^{\zeta}=B^{\zeta} / B$ is the $\zeta$-contravariant component of the unit vector $\mathbf{h}=\mathbf{B} / B$. The conventional relation between vector covariant and contravariant components $B_{\alpha}=g_{\alpha \beta} B^{\beta}$ is also used below. The physical poloidal component of velocity $U_{i \theta}$ is defined by the expression $U_{i \theta}=r V_{i}^{\theta}$, where $V_{i}^{\theta}$ is the $\theta$-contravariant component of the ion macroscopic velocity $\mathbf{V}_{i}$.

From Eqs. (15)-(17) we find the surface averaged ion heat flux,

$$
\Gamma_{T i}=-\frac{2 p_{i} \nu_{i} \cosh \eta}{M_{i} \omega_{c i}^{2}} \frac{\partial T_{i}}{\partial r}-\frac{5}{4} \frac{c B_{s} T_{i}}{\pi \varepsilon e_{i} R} \int_{0}^{2 \pi} d \theta \frac{n_{0}}{B^{2}} \frac{\partial T_{i}}{\partial \theta}
$$

Here, $n_{0}$ is the particle density.

Further, to find the poloidal rotation velocity $U_{i \theta}$ we employ the one fluid momentum equation

$$
M_{i} n_{0} \frac{d_{i} \mathbf{V}_{i}}{d t}=-\nabla p-\nabla \cdot \pi+\frac{1}{c}[\mathbf{j} \times \mathbf{B}]+\mathbf{F}^{h}
$$

where $p=p_{i}+p_{e}$ is the plasma pressure, $\boldsymbol{\pi}$ is the ion viscosity,${ }^{27}$ in which we take into account only parallel viscosity $\pi_{\|}$, therefore,

$$
\boldsymbol{\nabla} \cdot \hat{\boldsymbol{\pi}}=\frac{3}{2}\left\{[\mathbf{h}(\boldsymbol{\nabla} \cdot \mathbf{h})+(\mathbf{h} \cdot \boldsymbol{\nabla}) \mathbf{h}] \pi_{\|}+\mathbf{h}(\mathbf{h} \cdot \boldsymbol{\nabla}) \pi_{\|}-\frac{1}{3} \boldsymbol{\nabla} \pi_{\|}\right\},
$$

and $\mathbf{F}^{h}$ is the radio frequency force affecting electrons and ions. Using the ambipolarity condition,

$$
\int \boldsymbol{\nabla} \cdot \mathbf{j} d \mathbf{r}=\int_{0}^{2 \pi} \int_{0}^{2 \pi} j^{r} \sqrt{g} d \theta d \zeta \approx 0,
$$

which can be obtained from the current continuity equation $\boldsymbol{\nabla} \cdot \mathbf{j} \approx \mathbf{0}$ averaged over the magnetic surface, one obtains from Eq. (19)

$$
\begin{gathered}
-\left\langle\frac{\partial p}{\partial \theta}+(\boldsymbol{\nabla} \cdot \boldsymbol{\pi})_{\theta}+M_{i}\left(n_{0} \frac{d_{i} \mathbf{V}_{i}}{d t}\right)_{\theta}\right\rangle+r F_{\theta p}^{h} \approx 0 \\
\mu_{i \zeta} U_{i \zeta} \approx F_{\zeta p}^{h} .
\end{gathered}
$$

Here, $\mu_{i \zeta}$ is the coefficient describing the ion toroidal or anomalous viscosity or friction with neutrals, and $F_{\theta p}^{h}$ and $F_{\zeta p}^{h}$ are "physical" poloidal and toroidal components of the radio frequency force $\mathbf{F}^{h}$, respectively.

The plasma density poloidal perturbations $\widetilde{n}_{0}$ in Eq. (18) can be found from the momentum equation (19), taking into account the ion inertial terms connected with the subsonic toroidal flow, $U_{i \zeta}<c_{s}$, and the ion and electron temperature perturbations,

$$
\widetilde{n}_{0} \approx \frac{n_{0}\left(\widetilde{T}_{i}+\widetilde{T}_{e}\right)+\widetilde{\pi}_{\|}}{T_{i}+T_{e}}-\alpha \varepsilon^{*} n_{0} \cos \theta
$$

Using Eq. (24), the expression for the Cristoffel's symbol Eq. (14), and the parallel component of Eq. (19), one obtains from Eq. (22),

$$
\frac{1}{2 \pi r} \int_{0}^{2 \pi} d \theta\left\{\frac{3}{2} \widetilde{\pi}_{\|} \frac{\partial}{\partial \theta} \ln B-\alpha \varepsilon^{*}\left[n_{0}\left(\tilde{T}_{e}+\tilde{T}_{i}\right)+\widetilde{\pi}_{\|}\right] \sin \theta\right\}+F_{\theta p}^{h}=0
$$


Thus, our basic equations for the problem under study are Eqs. (18) and (25).

\section{Poloidally perturbed quanti- ties}

We see from Eqs.(18) and (25) that we should find the perturbed ion and electron temperatures and ion parallel viscosity. To find the particle temperatures, we proceed from the heat transport equations, ${ }^{27}$

$$
\frac{3}{2} n \frac{d_{\alpha} T_{\alpha}}{d t}+p_{\alpha} \boldsymbol{\nabla} \cdot \mathbf{V}_{\alpha}=-\boldsymbol{\nabla} \cdot \mathbf{q}_{\alpha}+Q_{\alpha},
$$

where $\alpha=i, e$, the heat exchange $Q_{\alpha}$ between ions and electrons is equal to

$$
Q_{i}=-Q_{e}=\frac{3 M_{e}}{M_{i}} n_{0} \nu_{e}\left(T_{e}-T_{i}\right)
$$

and particle heat fluxes are

$$
q_{i \|}=-3.91 \frac{n_{0} T_{i}}{M_{i} \nu_{i} q R} \frac{\partial T_{i}}{\partial \theta}, \quad q_{e \|}=-3.16 \frac{n_{0} T_{e}}{M_{e} \nu_{e} q R} \frac{\partial T_{e}}{\partial \theta}, \quad \mathbf{q}_{\alpha \perp}=\frac{5}{2} \frac{c n_{0} T_{\alpha}}{e_{\alpha} B}\left[\mathbf{h} \times \nabla T_{\alpha}\right] .
$$

Then, we obtain equations for perturbed quantities from Eqs. (26)-(28)

$$
\begin{aligned}
& -T_{i} \frac{U_{i \theta}}{r} \frac{\partial \tilde{n}}{\partial \theta}+\frac{1}{q R} \frac{\partial q_{i \|}}{\partial \theta}+\nabla \cdot \mathbf{q}_{i \perp}-\frac{3 M_{e} n_{0} \nu_{e}}{M_{i}}\left(\tilde{T}_{e}-\tilde{T}_{i}\right)=0, \\
& -T_{e} \frac{U_{e \theta}}{r} \frac{\partial \tilde{n}}{\partial \theta}+\frac{1}{q R} \frac{\partial q_{e \|}}{\partial \theta}+\nabla \cdot \mathbf{q}_{e \perp}+\frac{3 M_{e} n_{0} \nu_{e}}{M_{i}}\left(\tilde{T}_{e}-\tilde{T}_{i}\right)=0,
\end{aligned}
$$

The terms with the poloidal velocities $U_{i \theta}$ and $U_{e \theta}$ in Eqs. (29) and (30) can be obtained using the quasistationary continuity equations

$$
n_{j} \boldsymbol{\nabla} \cdot \mathbf{V}_{j}+\mathbf{V}_{j} \cdot \nabla n_{j}=0
$$

and the frozen-in condition

$$
\nabla \times\left[\mathbf{V}_{i} \times \mathbf{B}\right] \approx 0
$$

As a result, we have

$$
\tilde{V}_{i}^{\zeta} \approx q \tilde{V}_{i}^{\theta}, \quad \frac{\partial \tilde{V}_{i}^{\theta}}{\partial \theta}=-\frac{U_{i \theta}}{r} \frac{\partial}{\partial \theta} \ln \left(n_{0} \sqrt{g}\right)
$$

Using equation (33) and solving the system of Eqs.(29) and $(30)$, we find the perturbed particle temperatures

$$
\begin{gathered}
\tilde{T}_{i}=0.51 \frac{\varepsilon^{*} b T_{0}}{r \nu_{i} d(b)}\left\{\alpha U_{i \theta}\left(1+7.6 b \frac{M_{e}}{M_{i}}\right)-5 U_{T i}\left(1+\frac{\alpha}{2}\right)-\right. \\
\left.-3.8 \alpha b \frac{M_{e}}{M_{i}}\left(\frac{\varepsilon}{q} \frac{j_{\|}}{e_{i} n_{0}}+U_{p}\right)\right\} \sin \theta-\frac{0.32 \varepsilon^{*} b A T_{0}}{r \nu_{i} d_{1}(b)} U_{T i} \sin 2 \theta, \\
\tilde{T}_{i}+\tilde{T}_{e}=0.51 \frac{\varepsilon^{*} b T_{0}}{r \nu_{i} d(b)}\left\{\alpha U_{i \theta}\left(1+15.2 b \frac{M_{e}}{M_{i}}\right)-5 U_{T i}\left(1+\frac{\alpha}{2}\right)-\right. \\
\left.-7.6 \alpha b \frac{M_{e}}{M_{i}}\left(\frac{\epsilon}{q} \frac{j_{\|}}{e_{i} n_{0}}+U_{p}\right)\right\} \sin \theta-\frac{0.32 \varepsilon^{*} b A T_{0}}{r \nu_{i} d_{1}(b)} U_{T i} \sin 2 \theta,
\end{gathered}
$$

where $d(b)=1+2.2 b \sqrt{M_{e} / M_{i}}, d_{1}(b)=1+0.54 b \sqrt{M_{e} / M_{i}}$, and $b=q^{2} R^{2} / \lambda_{i}^{2}$. We have also used

$$
U_{e \theta}=U_{i \theta}-\frac{\epsilon}{q} \frac{j_{\|}}{e_{i} n_{0}}-U_{p}, \quad U_{p}=\frac{1}{M_{i} n_{0} \omega_{c i}} \frac{\partial p}{\partial r}, \quad U_{T i}=\frac{1}{M_{i} \omega_{c i}} \frac{\partial T_{0}}{\partial r}, \quad \nu_{e}=\nu_{i} \sqrt{\frac{2 M_{i}}{M_{e}}}
$$


with $T_{e 0} \approx T_{i 0}=T_{0}$. The expression for $U_{e \theta}$ in Eq. (36) can be obtained from momentum equations for electrons and ions. ${ }^{27}$

We also need to calculate the parallel viscosity $\tilde{\pi}_{\|}$ in Eqs. (24) and (25). Note, that the Braginskii's approach $^{27}$ for viscosity (the Navier-Stokes type of the viscosity) is not relevant for problems to be under study in this paper. Therefore, we take the parallel viscosity $\pi_{\|}$in the form derived in Refs. 26, 32, and 33. This type of viscosity is the so-called Burnett type of the viscosity. ${ }^{34}$ Besides the spatial derivatives of the ion velocity, it also includes the derivatives of the ion thermal fluxes $\mathbf{q}_{i}$. These additional terms are similar to the thermal force terms in the ion-electron friction in the momentum equation. ${ }^{27}$ Thus, we have, ${ }^{26,32,33}$

$$
\tilde{\pi}_{\|}=-\frac{2}{3} \frac{p_{i}}{\nu_{i}}(0.96 \beta-0.59 \gamma)
$$

where

$$
\begin{gathered}
\beta=3\left\{\mathbf{h}(\mathbf{h} \cdot \boldsymbol{\nabla}) \mathbf{V}_{i}+\frac{2}{5 p_{i}} \mathbf{h}(\mathbf{h} \cdot \boldsymbol{\nabla}) \mathbf{q}_{i}-\frac{1}{3}\left(\boldsymbol{\nabla} \cdot \mathbf{V}_{i}+\frac{2}{5 p_{i}} \boldsymbol{\nabla} \cdot \mathbf{q}_{i}\right)\right\}, \\
\gamma=-\frac{6}{5}\left\{\mathbf{h}(\mathbf{h} \cdot \boldsymbol{\nabla})\left(\mathbf{q}_{i}+0.27 \mathbf{q}_{i \|}\right)+\frac{1}{3}\left(\boldsymbol{\nabla} \ln p_{i} \cdot \mathbf{q}_{i}-\boldsymbol{\nabla} \cdot\left(\mathbf{q}_{i}+0.27 \mathbf{q}_{i \|}\right)\right)\right\} .
\end{gathered}
$$

Using Eqs.(28) and (33), we simplify Eqs. (38) and (39)

$$
\begin{gathered}
\beta=\frac{3}{r}\left\{-U_{i \theta} \frac{\partial}{\partial \theta} \ln \left(\sqrt{g} n^{2 / 3} B\right)+\frac{1}{q^{2} R^{2}} U_{i \theta} \frac{\partial g_{22}}{\partial \theta}+U_{T i} \frac{\partial}{\partial \theta} \ln \frac{B}{n}\right\}, \\
\gamma=-\frac{3}{r}\left\{0.34 U_{i \theta} \frac{\partial}{\partial \theta} \ln n+U_{T i}\left(1.36 \frac{\partial}{\partial \theta} \ln B-0.84 \frac{\partial}{\partial \theta} \ln n\right)\right\} .
\end{gathered}
$$

Finally, we obtain the expression for the parallel viscosity Eqs. (37), (40), and (41), employing Eqs. (8), (11), and $(24)$,

$$
\begin{aligned}
\widetilde{\pi}_{\|}= & \frac{1.92 \varepsilon^{*} p_{i}}{r \nu_{i}}\left\{U_{i \theta}[(1+0.19 \alpha) \sin \theta+0.5 A \sin 2 \theta]+\right. \\
& \left.+U_{T i}[(1.83+1.52 \alpha) \sin \theta+0.92 A \sin 2 \theta]\right\} .
\end{aligned}
$$

This expression gives us the possibility to find the ion ambipolar poloidal velocity $U_{i \theta}$.

\section{Ion fluxes and their analyses}

Now, we can derive the final expressions for plasma rotation velocity and the radial ion heat flux and analyze them. The poloidal velocity $U_{i \theta}$ can be obtained from Eqs. (25), (35), and (42),

$$
U_{i \theta}=G_{u 1}(\alpha, A) U_{T i}+G_{u 2}(\alpha, A)\left(\frac{\epsilon}{q} \frac{j_{\|}}{e_{i} n_{0}}+U_{p}\right)+1.39 \frac{\nu_{i} r^{2}}{\varepsilon^{* 2} M_{i} n_{0} v_{T i}^{2}} G_{u 3}(\alpha, A) F_{\theta}^{h},
$$

where

$$
\begin{gathered}
G_{u 1}(\alpha, A)=-\frac{f_{2}(\alpha, A)}{f_{1}(\alpha, A)}, \quad G_{u 2}(\alpha, A)=1.35 \frac{M_{e}}{M_{i}} \frac{\alpha^{2} b^{2}}{f_{1}(\alpha, A)}, \quad G_{u 3}(\alpha, A)=\frac{d(b)}{f_{1}(\alpha, A)}, \\
f_{1}(\alpha, A)=d(b)\left[\left(1+\frac{2}{3} \alpha\right)(1+0.19 \alpha)+0.25 A^{2}\right]+0.18 \alpha^{2} b\left(1+15.2 b \frac{M_{e}}{M_{i}}\right) \\
f_{2}(\alpha, A)=d(b)\left[\left(1+\frac{2}{3} \alpha\right)(1.83+1.52 \alpha)+0.46 A^{2}\right]-0.88 \alpha b\left(1+\frac{\alpha}{2}\right) .
\end{gathered}
$$


To plot functions $G_{u 1}(\alpha, A), G_{u 2}(\alpha, A), G_{u 3}(\alpha, A)$ in Fig. 1, we choose the parameter $b=50$. One can observe that function $G_{u 1}(\alpha, A)$ (figure 1), as in the case of an axially-symmetric tokamak, ${ }^{30}$ changes sign at $\alpha_{0} \approx 2 d(b) / b$. This quantity coincides with our previously obtained results. ${ }^{26,28-31}$ It is a very interesting and important result that inertial forces, affecting ions, enforce ion poloidal rotation velocity $U_{i \theta}$ to change its sign in the absence of radio frequency waves in a plasma. This effect, which was originally obtained in Ref. 26, is also relevant for weakly collisional plasmas but sign changing takes place at the Mach number of the order unity. ${ }^{35}$ The maximum of function $G_{u 1}(\alpha, A)$ occurs at $A \approx 0$ and is $G_{u 1}(\alpha, 0) \approx 2.4$. Increasing the parameter $A$, the maximum of the quantity $G_{u 1}(\alpha, A)$ decreases and shifts to supersonic values, $\alpha>1$. Nevertheless, only the case $\alpha<1$ is of the practical interest.

The function $G_{u 2}(\alpha, A)$ characterizes the role of the parallel current and diamagnetic drift in the ion poloidal rotation velocity. This function increases with the parameter $\alpha$, but stays at a small level,
$G_{u 2}(\alpha, A)<<1$ for $\alpha<1$.

The function $G_{u 3}(\alpha, A)$ describes the role of external forces in inducing the ion poloidal rotation. This function decreases with the growth of the plasma toroidal rotation, and, correspondingly, so does the role of external forces on the ion poloidal rotation. The induced radial electric field, which is currently supposed to be the dominant quantity for transport barrier creation in tokamaks, ${ }^{12-17}$ can be found using the expression which is defined via the ion velocity from the ion motion equation, ${ }^{27}$

$$
\begin{gathered}
E_{r} \approx \frac{B}{c}\left(U_{i \zeta} h_{\theta p}+U_{p i}-U_{i \theta}\right), \\
U_{p i}=\frac{1}{M_{i} n_{0} \omega_{c i}} \frac{\partial p_{i}}{\partial r}
\end{gathered}
$$

Using Eqs. (11), (24), (34), (42), and (43), we derive the magnetic surface averaged radial ion heat flux from Eq. (18). It is convenient to write the final equation in the Shafranov form, ${ }^{24,25}$

where

$$
\begin{gathered}
\Gamma_{T i}=-\frac{2 n T_{i} \nu_{i}}{M_{i} \omega_{c i}^{2}} \frac{\partial T_{i}}{\partial r}\left\{\cosh \eta+1.6 q^{2} \frac{\varepsilon^{* 2}}{\varepsilon^{2}}\left[G_{T 1}(\alpha, A)+G_{T 2}(\alpha, A) \frac{1}{U_{T i}}\left(\frac{\epsilon}{q} \frac{j_{\|}}{e_{i} n_{0}}+U_{p}\right)-\right.\right. \\
\left.\left.-1.39 \frac{\nu_{i} r^{2}}{\varepsilon^{* 2} U_{T i} M_{i} n_{0} v_{T i}^{2}} G_{T 3}(\alpha, A) F_{\theta}^{h}\right]\right\},
\end{gathered}
$$

In a supposition that the collisional parameter $b$ satisfies the inequality $1<b<\sqrt{M_{i} / M_{e}}$, one obtains the well-known result for the radial ion heat flux. ${ }^{24,25}$

The coefficients $G_{T 1}(\alpha, A)$ and $G_{T 3}(\alpha, A)$ are plotted in Fig. 2. The quantity $G_{T 2}(\alpha, A)$ is small under the condition $b \leq M_{i} / M_{e}$, therefore, it is not presented in Fig. 2. The functions $G_{T 1}(\alpha, A)$ and $G_{T 3}(\alpha, A)$ characterize the neoclassical contribution and the influence of external forces, respectively. The function $G_{T 1}(\alpha, A)$ is a growing function of the elongation $A$ and the squared Mach number $\alpha$. The role of external forces increases with the Mach number, nevertheless, less intensively for larger elongations [see the function $G_{T 3}(\alpha, A)$ in Fig. 2].

\section{Conclusion}

We have studied the effect of plasma subsonic toroidal flows induced by Alfvén waves on poloidal rotation and ion heat conductivity of collisional plasmas of elongated tokamaks with smooth profiles of macroscopic plasma parameters. This study is important as ion neoclassical 
fluxes are sometimes in a satisfactory coincidence with the experimental results, ${ }^{36}$ as opposed to the electron

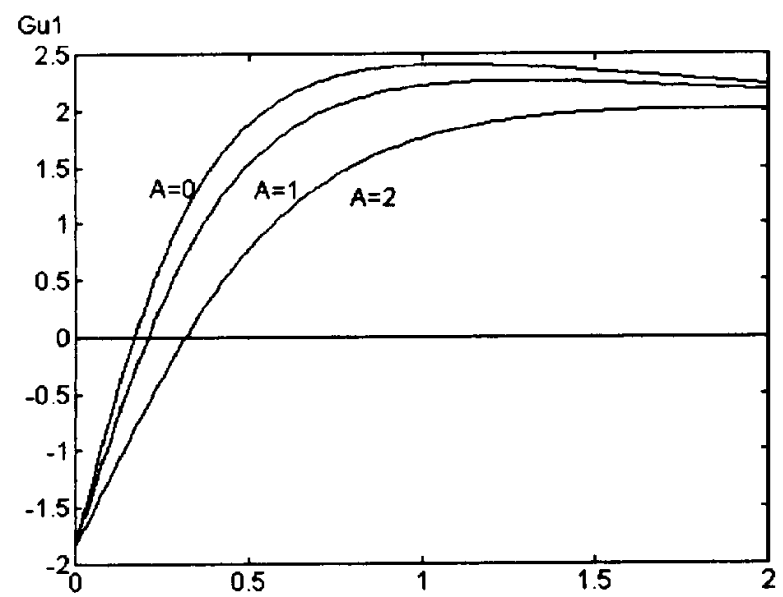

$\alpha$

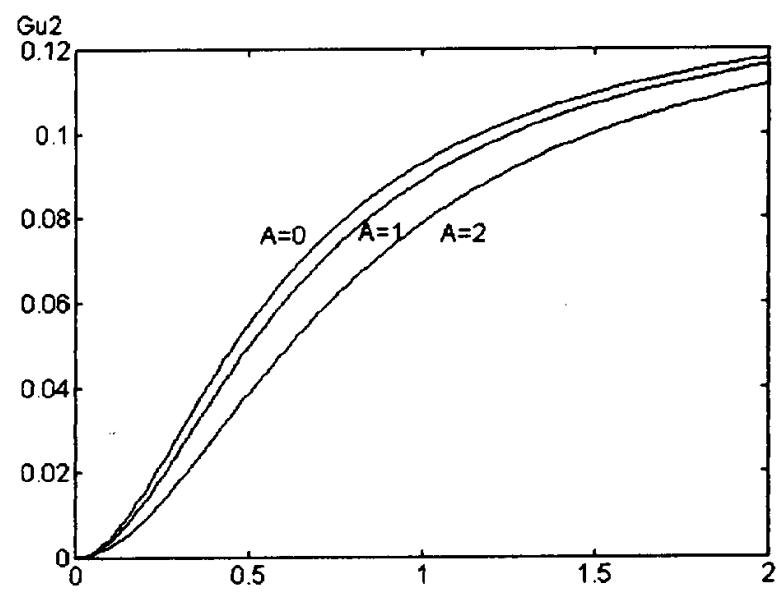

$\alpha$

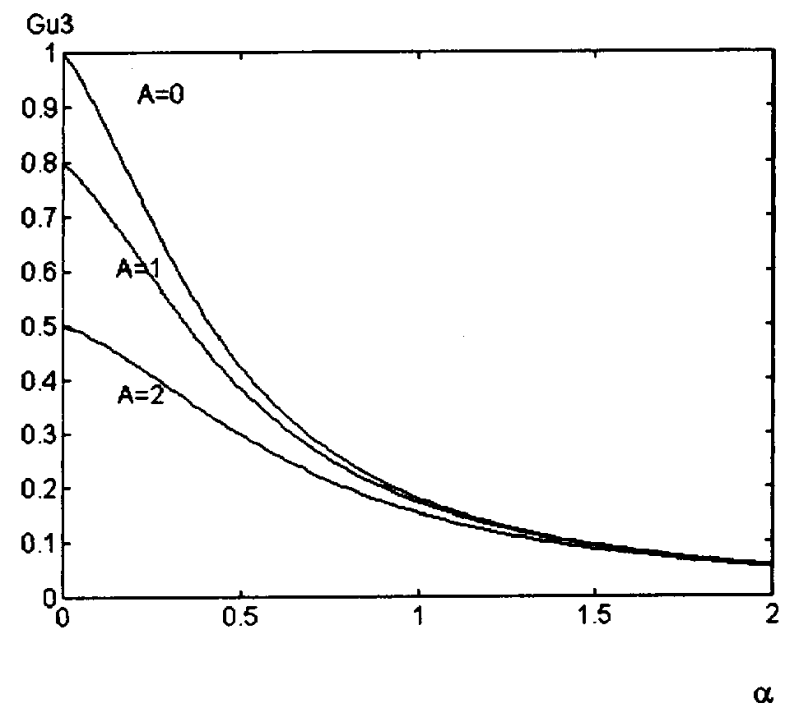

Figure. 1 The dependence of the functions $G_{u 1}(\alpha, A)$, $G_{u 2}(\alpha, A)$, and $G_{u 3}(\alpha, A)$ on $\alpha$, for different magnitudes of the parameter $A: A_{1}=0, A_{2}=1, A_{3}=2$.
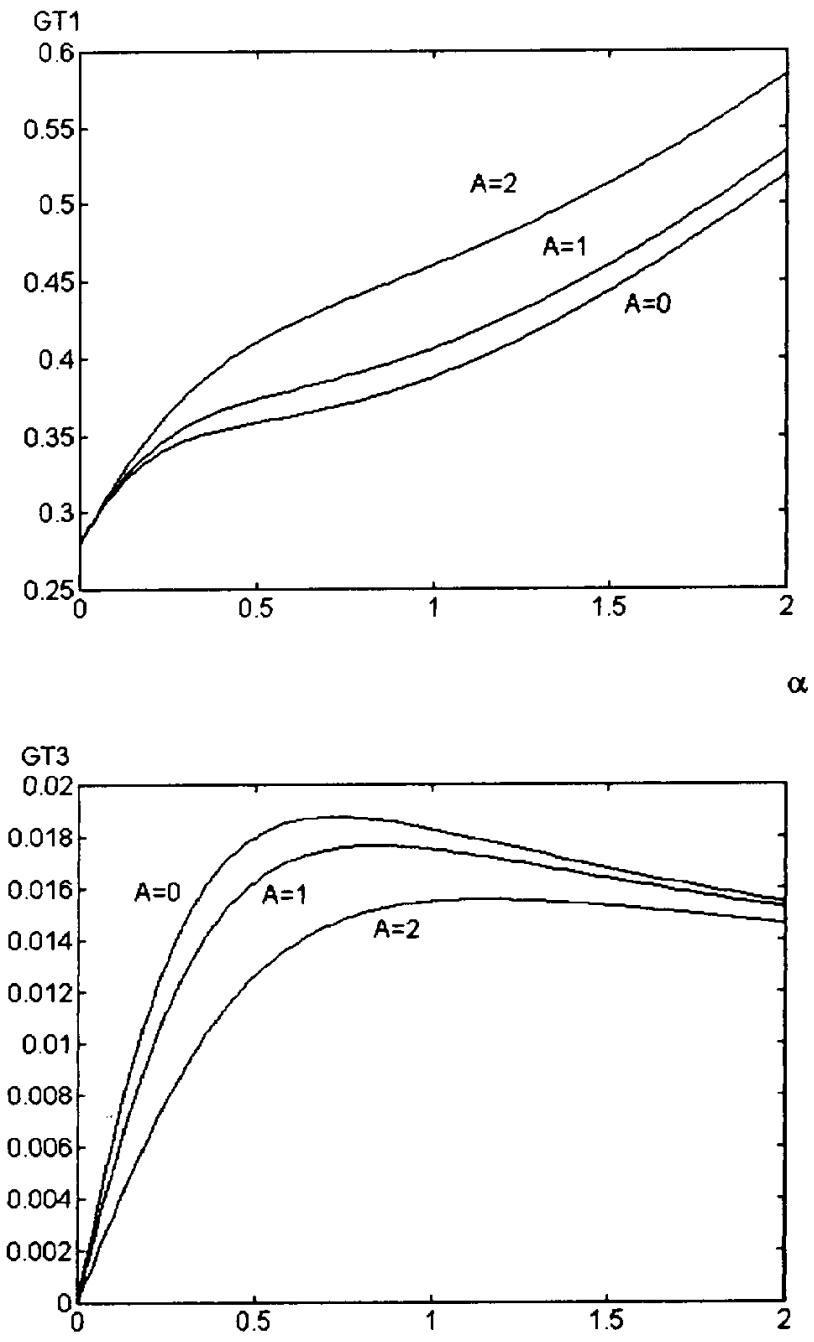

$\alpha$

Figure. 2 The dependence of the functions $G_{T 1}(\alpha, A)$ and $G_{T 3}(\alpha, A)$ on $\alpha$, for different magnitudes of the parameter $A: A_{1}=0, A_{2}=1, A_{3}=2$.

anomalous transport. This paper is a generalization of our previous results for the case of elongated tokamaks. ${ }^{26,28-31}$ It is shown that a tokamak elongation can substantially affect ion neoclassical fluxes. These fluxes are presented in the paper as functions of three quantities to be proportional to 1) the conventional ion temperature gradient, 2) the parallel current and diamagnetic drift, and 3) radio frequency forces. The proper coefficients are plotted on the Fig. 1 and Fig. 2. Once more, the importance of the collisional parameter $b$ is emphasized in the paper (see also Refs. 21-23, 26, 28-31). It is also demonstrated that the very interesting effect of changing the sign of the ion poloidal rotation velocity in collisional tokamak plasmas, which depends on the square of the Mach number $\alpha$, obtained previously, ${ }^{26}$ also takes place in elongated tokamaks. The poloidal velocity has a maximum as a function of the Much number. This sign changing and maximum 
of the ion poloidal rotation is the consequence of taking into account inertial forces affecting ions. It is also shown that the radio frequency forces can in some cases determine the ion neoclassical fluxes in elongated tokamaks.

\section{Acknowledgments}

This work was supported by the Research Support Foundation of the State of São Paulo (FAPESP), National Council of Scientific and Technological Development $(\mathrm{CNPq})$, and Excellence Research Programs (PRONEX) RMOG 50/70 grant from the Ministry of Science and Technology, Brazil.

\section{References}

[1] Ya. I. Kolesnichenko, V. V. Parail, and G. V. Pereverzev, in Reviews of Plasma Physics, edited by B. B. Kadomtsev, (Consultants Bureau, New York, 1990) Vol. 17, p. 3.

[2] A. G. Elfimov, A. G. Kirov and V. P. Sidorov, in HighFrequency Plasma Heating, edited by A. G. Litvak (American Institute of Physics Translation series, NewYork, 1992), p.239.

[3] R. Klima, Czech. J. Phys. B 18, 1280 (1968).

[4] N. J. Fish and F. F. Karney, Phys. Fluids 24, 27 (1981).

[5] D. W. Faulconer, Fusion Technology 33 (2T) 219 (1998).

[6] R.A.Demirkhanov, A.G.Kirov, L.F.Ruchko, and A.V.Sukachev, Soviet Phys. JETP Lett. 33, 28 (1981).

[7] G. A. Collins, F. Hofmann, B. Joye, R. Keller, A. Lietti, J. B. Lister, and A. Pochelon, Phys. Fluids, 29, 2260 (1986).

[8] N. Hershkovitz, P. Moroz, P. Probert, E. Y. Wang, T. Intrator, D. Diebold, R. Breun, D. Brouchous, M. Doczy, D. Edgel, A. Elfimov, R. Fonk, M. Kishinevsky, C. Litwin, P. Nonn, S. Regan, J. Sorensen, J. Tataronis, M. Vucovich, X. Wang, and S. Wukitch, in Proceedings of the 15th International Atomic Energy Agency Conference on Plasma Physics and Controlled Fusion, Seville, 1994 (International Atomic Energy Agency, Vienna, 1995), Vol. 2, p. 245.

[9] I. C. Nascimento, R. M. O.Galvão and TCABR team, in Proc. of X Intern. Conf. on Plasma Physics. Contr. Papers. Brazil, Foz do Iguaçu, 31.10.94-04.11.94, (AIP, Woodbury, New York, 1996), Vol. 1, p. 69.

[10] R. M. O. Galvão, A. G. Elfimov, G. AmaranteSegundo, V. S. Tsypin, L. F. Ruchko, I. C. Nascimento, M. Tendler. Plasma Phys. Contr. Fusion 41, A487 (1999).

[11] G. G. Craddock and P. H. Diamond, Phys. Rev. Lett. 67, 1535 (1991).
[12] V. S. Tsypin, A. G. Elfimov, M. Tendler, C. A. de Azevedo, and A. S. de Assis, Phys. Plasmas 5, 7 (1998).

[13] V. S. Tsypin, R. M. O. Galvão, I. C. Nascimento, A. G. Elfimov, M. Tendler, C. A. de Azevedo, and A. S. de Assis, Phys. Rev. Lett. 81, 3403 (1998).

[14] V. S. Tsypin, I. C. Nascimento, R. M. O. Galvão, A. G. Elfimov, G. S. Amarante Segundo, and M. Tendler Phys. Plasmas 6, 3548 (1999).

[15] H. Biglary, P. H. Diamond, and P. W. Terry, Phys. Fluids B 2, 1 (1990).

[16] T. S. Hahm and K. H. Burrel. Phys. Plasmas 2, 1648 (1995)

[17] K. H. Burrell, Phys. Plasmas 4, 1499 (1997).

[18] H. L. Berk, A. A. Galeev. Phys. Fluids 10, 44 (1967).

[19] K. C. Shaing and R. D. Hazeltine, Phys. Fluids B 4, 2547 (1992).

[20] S. Wukitch, C. Litwin, M. Harper, R. Parker, and N. Hershkovitz, Phys. Rev. Lett. 77, 294 (1996).

[21] A. Rogister, Phys. Plasmas 1, 619 (1994).

[22] A. Rogister, Phys. Rev. Lett. 81, 3663 (1998).

[23] A. Rogister, Phys. Plasmas 6, 200 (1999).

[24] V. D. Shafranov, Sov. Atom. Energy 19, 1008 (1965).

[25] V. D. Shafranov, J. W. Connor, and C. J. Watson, Sov. J. Plasma Phys. 2, 99 (1976)

[26] A. B. Mikhailovskii and V. S. Tsypin, Sov. Phys.-JETP 56, 75 (1982).

[27] S. I. Braginskii, in Reviews of Plasma Physics, edited by M. A. Leontovich (Consultants Bureau, New York, 1965), Vol. 1, p. 205.

[28] V. S. Tsypin, Sov. J. Plasma Phys. 11, 661 (1985).

[29] V. S. Tsypin, Sov. J. Plasma Phys. 14, 466 (1988).

[30] V. S.Tsypin, D. Kh.Morozov, J. J. E.Herrera, J. J. Martinell, M. Tendler, I. F. Potapenko, A. S. de Assis, and C. A. de Azevedo. Plasma Phys. Control. Fusion 39, 1681 (1997).

[31] V. S. Tsypin, C. A. de Azevedo, and A. S. de Assis, Physics Letters A. 219, 282 (1996).

[32] A. B. Mikhailovskii and V. S. Tsypin, Plasma Physics 13, 785 (1971).

[33] A. B. Mikhailovskii and V. S. Tsypin, Beiträge Plasma Physik 24, 335 (1984).

[34] D. Burnett, Proc. Lond. Math. Soc. 39 385; 40382 (1935).

[35] S. K. Wong and K. H. Burrel, Phys. Fluids 251863 (1982).

[36] F. Wagner and U. Strouth, Plasma Phys. Contr. Fusion 341803 (1992). 